\title{
Efficient and Robust Traction Laws for the Modeling of Adhesively Bonded Joints
}

\author{
Peter A. Gustafson*, Anthony M. Waas ${ }^{\dagger}$ \\ Department of Aerospace Engineering, University of Michigan, Ann Arbor, MI 48109, USA
}

\begin{abstract}
A discrete cohesive zone method finite element is used to evaluate traction law efficiency and robustness in predicting decohesion in a finite element model. Three traction laws are reported and are compared from the perspective of their computational efficiency and robustness. The smooth traction laws (based on the beta distribution and sine functions) are found to have greater computational efficiency than the trapezoidal traction law. Efficiency gains are due to the elimination of the stiffness discontinuities associated with the generalized trapezoidal traction law. The sinusoidal traction law is found to be more robust and efficient than the other traction laws.
\end{abstract}

\section{Introduction}

TINITE element modeling and other forms of computational analysis have become indispensable tools in $\mathrm{F}$ system design and mission preparation. An active area of research is the application of these methods to the field of adhesive systems, bonded joints, and delamination. Though finite element (FE) modeling of adhesively bonded joints began as early as $1971,{ }^{1,2}$ the field is not yet mature. Recent efforts include the following techniques: continuous cohesive zone method, ${ }^{3-7}$ discrete cohesive zone method, ${ }^{8-15}$ virtual crack closure technique, ${ }^{16-22}$ element internal cohesive segments, ${ }^{23}$ and other adhesive region models. ${ }^{24-27}$ The references provided here are not an exhaustive list.

The continuous cohesive zone method (CCZM) and discrete cohesive zone method (DCZM) models are particularly well suited to analysis of decohesion in composite materials. The length scale associated with the process zone is larger than any characteristic length of the material. ${ }^{28-34}$ Cohesive zone models have begun to be incorporated into commercial software including Abaqus $\AA^{35,36}$ and Genoa ${ }^{37}$ as well as freely available research codes like Tahoe® ${ }^{38}$

The DCZM technique is seen as a promising alternative to the CCZM. Continuous cohesive zone elements have been found to be mesh sensitive (in some circumstances), to suffer from convergence difficulty during the softening portion of the cohesive law, and to have sensitivity to aspect ratio. ${ }^{23,39-41} \mathrm{~A}$ rich description of the strengths and weaknesses of the cohesive zone methodologies is provided by Xie and Waas. ${ }^{13}$ In contrast, the DCZM methodology treats the process zone as a point-wise spring foundation that is discretized to node pairs of adjoining surfaces. The method is scalable to the node spacing and is claimed to be free of mesh dependency. ${ }^{8,13}$ The stiffness matrix is sparse and is therefore computationally efficient. Though it does not avoid instability due to strain softening, careful application of damping stabilization can improve convergence.

Though they are an important advancement, these "production level" continuum cohesive elements have not been widely adopted. A primary obstacle to their widespread use is the local and highly non-linear constitutive response of the adhesive materials and their adherends. In addition, the analyst often encounters the physical phenomenon that a joint (poorly designed or by necessity) can be subject to catastrophic failure modes that are accompanied by large and sudden changes in load and structural stiffness. These two analytical obstacles, when coupled together, cause great difficulty in obtaining a converged solution. To date

*Graduate Student Research Assistant. Member AIAA. email: petegus@umich.edu

${ }^{\dagger}$ Professor. Associate Fellow, AIAA. email: dcw@umich.edu

Copyright (C) 2008 by Peter A. Gustafson. Published by the American Institute of Aeronautics and Astronautics, Inc. with permission. 
they have prevented widespread deployment of the available analysis techniques. There are ongoing efforts to develop improvements to the available methods. ${ }^{42-44}$

Using simple and elegant arguments, Hillerborg et al. ${ }^{8}$ proposed the essential components of a springbased traction law element capable of analyzing crack formation and propagation. The "fictitious crack" element featured the ability to predict new cracks based on a stress criterion $\left(\sigma_{\mathrm{c}}\right)$ while also predicting crack growth based on an energy criterion $\left(G_{\mathrm{c}}\right)$. The concept has experienced a (independently conceived) revival and found application to laminated composite materials,,${ }^{9,10}$ geometrically non-linear behavior, ${ }^{14}$ and Mode II fracture, ${ }^{13}$ and has recently been referred to as the DCZM. Similar elements have also been presented. ${ }^{45,46}$

Reliable and efficient convergence remains the largest computational hurdle in deployment of robust cohesive models. The propagation of a softening law through a structure is computationally challenging. ${ }^{47}$ Methods to address this difficulty have had mixed success. Arc length methods have been attempted by several authors, $, 48,49$ however they tend to suffer from large spurious oscillations. It was reported that this was due to a lack of mesh refinement, however it is unclear what role the strain softening traction law may have played in the oscillations. Line search algorithms are often considered a remedy for lack of convergence, however complications arise for non-conservative problems. ${ }^{50}$ When used in conjunction with the primary methods of this work, neither of these methods were found to have significant additional benefits.

The present approach to obtain robust convergence is to apply a traction law with "smooth" stiffness gradients while simultaneously using viscous damping for stabilization when necessary. Others have employed "smooth" laws, though it does not appear that they were chosen with improved convergence as their primary goal. For example, Goyal et al. ${ }^{26}$ used a law based on the exponential function which therefore has no stiffness discontinuities. A line search algorithm was employed and the stiffness was set to zero when the law was undergoing strain softening. It was claimed that convergence difficulties were eliminated, however independent verification is still pending. Alfano and Crisfield ${ }^{48}$ claim that the use of the tangent modulus should give better convergence of the residual norms and this method was used for the bulk of the current work. Corigliano et al. ${ }^{51}$ also used an exponential traction law, however no claim was made about convergence.

In this article, a DCZM element and several traction laws are evaluated from the perspective of efficiency and robustness for use in modeling decohesion in structures. The element features a computationally efficient traction separation formulation, optional viscous damping for stabilization, standard 2D and 3D interfaces, and a modular interface for specifying the desired traction law. Three traction laws are presented, one of which is commonly used and the others which are designed specifically for convergence efficiency. A comparison of the convergence efficiency and robustness of the three laws is provided based on models of two coupon-level tests.

\section{Solution efficiency}

In Gustafson and Waas, ${ }^{15}$ it was reported that crack stability depends on the specimen loading and the traction law. A more detailed description of this dependency is reported in this section. Two topics must be considered when addressing solution efficiency for the computation of cohesive problems: structural instability and numerical convergence.

\section{A. The critical crack separation}

In structures that can decohere, structural instability primarily arises from the sudden failure of the bonded interface. In this case, the absence of a formulation accounting for kinetic energy can cause an imbalance between the strain energy release rate and the energy dissipation due to permanent deformation. This imbalance causes a sudden and large change in the stiffness and load of the system. As a result, an incremental FE solver can struggle to find an equilibrium solution.

At a given level of strain energy release rate, the structural stability margin is primarily dependent on the critical crack separation $\left(\delta_{\mathrm{c}}\right)$ in figures $(1,2$, and 4$)$. If the value of $\delta_{\mathrm{c}}$ is large, the system is "soft". For a given displacement in this state, load transfer occurs over a large material volume and the strain energy is relatively small for the global displacement. As the global displacement increases, the loads are "smoothly" transitioned to neighboring elements via a gradual and dispersed stiffness change. The solver finds the equilibrium path with relative ease. Conversely, if $\delta_{\mathrm{c}}$ is small, the load transfer is concentrated 
into a small material volume with a relatively large strain energy. An increase in global displacement causes a very localized change in stiffness and displacement field and it is more difficult for the solver to find the equilibrium path.

Based on these arguments, larger values of $\delta_{\mathrm{c}}$ result in more efficient solutions due to ease of convergence.

\section{B. The stiffness gradient}

A second consideration in solution efficiency is the continuity of the traction law. Consider the Newton solver as described by Simulia. ${ }^{35}$ Linearization of the virtual work equations yields:

$$
F^{N}\left(u_{i}^{M}+c_{i+1}^{M}\right)=0
$$

$F^{N}$ is the force component conjugate to the $N$ th variable in the problem. $u_{i}^{M}$ and $c_{i+1}^{M}$ are the values of the $M$ th variable in increment $i$ and the absolute error of the $M$ th variable in increment $i+1$. A Taylor expansion of Eq. (1) gives:

$$
F^{N}\left(u_{i}^{M}\right)+\frac{\partial F^{N}\left(u_{i}^{M}\right)}{\partial u_{i}^{P}} c_{i+1}^{P}+\frac{\partial^{2} F^{N}\left(u_{i}^{M}\right)}{\partial u_{i}^{P} \partial u_{i}^{Q}} c_{i+1}^{P} c_{i+1}^{Q}+\ldots=0 .
$$

If the force functions are sufficiently smooth and $u_{i}^{M}$ is a close approximation of the true solution, the higher order terms in Eq. (2) are negligible. The value of the incremental error (the correction to the true displacement) can be iteratively computed via:

$$
K_{i}^{N P} c_{i+1}^{P}=-F_{i}^{N}
$$

where $K_{i}^{N P}$ is the tangent stiffness matrix defined by:

$$
K_{i}^{N P}=\frac{\partial F^{N}\left(u_{i}^{M}\right)}{\partial u^{P}}
$$

and the residual force vector is:

$$
F_{i}^{N}=F^{N}\left(u_{i}^{M}\right)
$$

Subsequent iterations are computed as:

$$
u_{i+1}^{M}=u_{i}^{M}+c_{i+1}^{M}
$$

until a converged solution is obtained.

In this scheme, the value of the correction is linearly computed from the current residual vector and the current tangent stiffness matrix. Convergence is accepted when the values of $F_{i}^{N}$ and $c_{i+1}^{M}$ are sufficiently small. However, if the higher order derivatives (the stiffness gradient) in Eq. (2) are large, then the higher order terms can be non-negligible. This causes Eq. (4) to compute a poor approximation for the correction vector, leading to convergence difficulties. When this occurs, it is necessary to reduce the solver increment size. The reduction results in higher computational cost. In this way, the smoothness of the traction law is a critical component of the solution efficiency and robustness.

\section{The traction laws}

The DCZM element described in Gustafson and Waas ${ }^{15}$ is modular in the application of traction laws. It has been shown that the form of the traction law is not critical in the global load displacement response. ${ }^{7,13,52-57}$ Therefore, the modularity of the DCZM element offers some flexibility in controlling different aspects of the cohesive model. More specifically, the form of the traction law can be specified to suit one of several purposes. For example, the law applied in the FE model can be "most accurate" representation of the actual traction separation response. The law can also be formulated for the simplicity of implementation, ${ }^{50}$ or for the purposes of numerical efficiency and robustness. In this section, these last two philosophies are adopted and three traction laws are reported and evaluated.

A feature of each implementation is that the element will unload following along a line from the origin to the force associated with the extreme separation. Reload follows the same path as the prior unloading path, preserving any material degradation. 


\section{A. The trapezoidal traction law}

The trapezoidal traction law (schematically shown in figure 1) is perhaps the most commonly used law. It is a generalization of the triangular law used in the Xie element ${ }^{13}$ and elsewhere. Furthermore, it is a convenient implementation due to the simplicity of formulating the three linear regions of the law. The three regions are referred to as the initial linear response region, the optional "plastic deformation" region, and the strain softening region.

Each fracture mode (I, II, III) requires three parameters to implement the trapezoidal traction law (TTL). In two dimensional problems, the required parameters are the critical energy release rates $\left(G_{\text {Ic }}, G_{\text {IIc }}\right)$, the critical stresses $\left(\sigma_{\text {Ic }}, \sigma_{\text {IIc }}\right)$, and the plasticity fractions $\left(\alpha_{\mathrm{pl}}^{I}, \alpha_{\mathrm{pl}}^{I I}\right)$. In the TTL, the "plastic" fraction (the rectangular area below the flat section of the traction law in figure 1) is a user controllable variable bound by zero (restoring a triangular law) and one.

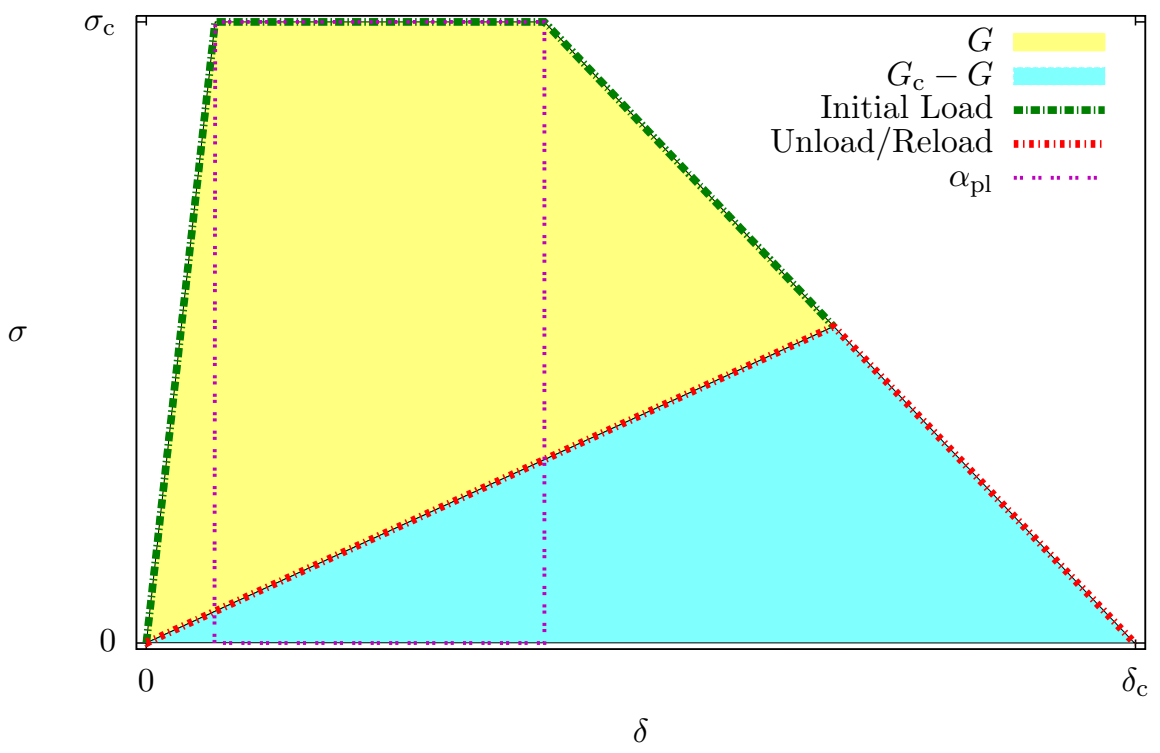

Figure 1: The trapezoidal traction law

\section{Efficiency considerations related to the TTL}

Despite being simple in implementation, the TTL is an example of a law that can suffer from severe convergence difficulties. For example, as the peak stress is crossed in the triangular law, the tangent stiffness suffers a change in sign from positive to negative. The stiffness gradient is infinite at that point, potentially ${ }^{\mathrm{a}}$ causing a significant decrease in the the increment size. These discontinuities were recognized by Alfano and Crisfield ${ }^{48}$ and were referred to as limit points. Furthermore, once the separation has passed the critical zone at a given integration point, large solution increments can be restored only if no other integration points are near their critical separation. When hundreds or thousands of discontinuous integration points are present, the solution can fail to converge or the average increment size can cause the analysis to be prohibitively expensive.

If plasticity is introduced into the TTL, the effect on convergence may be positive as the magnitude of the step stiffness change is reduced. However, in doing so a second step change in stiffness is necessarily introduced. Therefore, the overall effect can be either an improvement or a worsening of the computation efficiency.

Beyond the stiffness discontinuities, the efficiency of the TTL is also effected by the magnitude of the three parameters that are used to define it. The value of $\delta_{\mathrm{c}}$ is dependent on the cohesive strength $\left(\sigma_{\mathrm{c}}\right)$, the plasticity fraction $\left(\alpha_{\mathrm{pl}}\right)$, and the critical energy release rate $\left(G_{\mathrm{c}}\right)$. The efficiency is also effected by the

\footnotetext{
${ }^{a}$ It is common to use step changes in constitutive response in FE modeling and doing so does not always cause convergence difficulties. It is the magnitude of the step that is important as well as the number of integrations points that are actively transitioning.
} 
critical energy release rate $\left(G_{\mathrm{c}}\right)$ through the effects directly described by classical fracture mechanics and crack stability.

In order to improve the convergence efficiency, it is worth investigating the use of a "smooth" constitutive law which avoids the issues surrounding a discontinuous stiffness. Traction laws with "smooth" derivatives have been examined before, ${ }^{49,58}$ however the form of the law appears to be chosen for mathematical convenience instead of numerical considerations. Further, no quantitative assessment of the relative "convergence efficiency and robustness" of the laws were reported.

The objective of the remainder of this article is to find a traction law that also has a smooth derivative in order to have a more continuous stiffness matrix. Two smooth traction laws are proposed based on the beta distribution and sine functions.

\section{B. The beta distribution traction law}

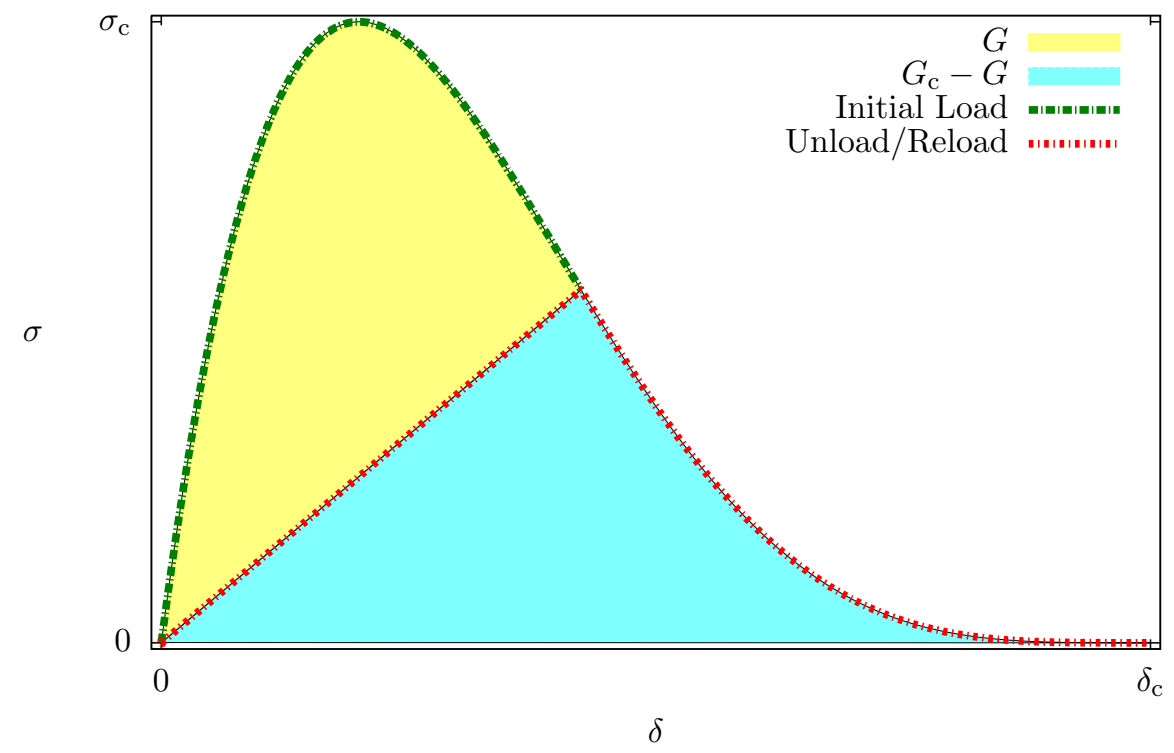

Figure 2: The beta distribution traction law.

Consider the beta probability distribution:

$$
\beta(x, a, b)=\frac{x^{a-1}(1-x)^{b-1}}{\int_{0}^{1} u^{a-1}(1-u)^{b-1} d u},
$$

where the denominator is the beta function. As a probability distribution, the value of its integral is one over the interval $x=[0,1]$.

$$
\int_{0}^{1} \beta(x, a, b) d x=1
$$

Therefore, the distribution can be mapped to a finite traction-separation space with known values of $G_{\mathrm{c}}$.

\section{Mapping the BDTL to the critical energy release rate}

Two parameters are required $\left(G_{\mathrm{c}}, \sigma_{\mathrm{c}}\right)$ and two requirements must be met to complete the mapping. First, the maximum value of $\beta(x, a, b)$ is mapped to the critical stress through a multiplicative constant. Subsequently, the traction law can be written as:

$$
\sigma_{\beta}\left(\frac{\delta}{\delta_{\mathrm{c}}}, a, b\right)=\frac{\sigma_{\mathrm{c}} \beta\left(\frac{\delta}{\delta_{\mathrm{c}}}, a, b\right)}{\beta_{\max }(a, b)} .
$$


In Eq. (9), $\beta_{\max }(a, b)$ is the maximum value of the probability density function (PDF). Second, the traction law must integrate to the value of $G_{\mathrm{c}}$.

$$
\int_{0}^{\delta_{\mathrm{c}}} \frac{\sigma_{\mathrm{c}}}{\beta_{\max }(a, b)} \beta\left(\frac{\delta}{\delta_{\mathrm{c}}}, a, b\right) d \delta=G_{\mathrm{c}}
$$

A change of variables is required to map the integral in Eq. (10) into the space of the PDF:

$$
d \delta=\delta_{\mathrm{c}} d x
$$

which allows Eq. (10) to be written as:

$$
\frac{\sigma_{\mathrm{c}} \delta_{\mathrm{c}}}{\beta_{\max }(a, b)} \int_{0}^{1} \beta(x, a, b) d x=G_{\mathrm{c}} .
$$

In Eq. (12), the integral portion of the left hand side evaluates to one, therefore the critical displacement is calculated as:

$$
\delta_{\mathrm{c}}=\frac{\beta_{\max }(a, b)}{\sigma_{\mathrm{c}}} G_{\mathrm{c}} .
$$

The final calculation is of the maximum value of the beta distribution $\left(\beta_{\max }(a, b)\right)$. After multiplying the right hand side of (7) by its denominator, one must extremize:

$$
(1-x)^{b-1} x^{a-1} .
$$

Taking the derivative of Eq. (14) with respect to $x$ and equating it to zero yields:

$$
(a-1)(1-x)^{b-1} x^{a-2}-(b-1)(1-x)^{b-2} x^{a-1}=0 .
$$

Eq. (15) can be solved for $x_{\max }$ which is a maximum for values of $a$ and $b$ that are appropriate for the beta distribution traction law (BDTL):

$$
x_{\max }=\frac{a-1}{b+a-2} .
$$

Inserting Eq. (16) into the distribution function, the maximum value is given by:

$$
\beta_{\max }(a, b)=\frac{x_{\max }^{a-1}\left(1-x_{\max }\right)^{b-1}}{\int_{0}^{1} u^{a-1}(1-u)^{b-1} d u} .
$$

With Eq. (17), the mapping of the traction law is complete. The BDTL, shown in figure 2, has been implemented as a traction law module to accompany the DCZM element created for Abaqus®.

\section{Efficiency considerations related to the BDTL}

It has been established that the form of the traction law affects the computational efficiency through the increment size. Specifically, it was shown that the second derivative of the traction law, when large, can cause difficulty is obtaining an accurate correction vector. Here it will be shown that the BDTL can ensure a smooth traction law.

The parameters $a$ and $b$ have a significant effect on the efficiency of the solution as well as the ability of the solution to obtain a converged equilibrium. In order for the traction law to be reasonable and resemble the more commonly used laws, the appropriate ranges are $1<a<3$ and $a<b<10$. These values are not all appropriate for use in an element, where the primary criteria for appropriateness are the resulting stiffness gradients and the overall shape of the distribution.

As shown in the figure 3(a), low values of $a$ skew the beta distribution to the left. High values of $b$ do so also, but with the additional effect of causing an increase in concavity on the down slope of the curve. At first glance, it would appear that a value of $a$ just larger than 1.0 would closely match the traditional triangular traction law while providing a continuous derivative.

However, the further to the left the curve is skewed, the more difficult it is to obtain a converged solution due to larger stiffness gradients. Similarly, the system itself is less stable since smaller portions of the 
structure are transferring load, resulting in a higher energy release rate. Beyond generalities, figure 3(c) shows that the stiffness gradients near $x=0$ approach infinity as $a$ approaches 1.0. For $a$ greater than 2.0, the stiffness gradients remain large around the origin, where the stiffness starts at zero. Figures $3(\mathrm{~b})$ and $3(\mathrm{~d})$ examine the effect of small variations around the value $a=2.0$ and show that even small diversions cause significant stiffness gradients. Therefore, $a=2.0$ is most appropriate for this traction law. ${ }^{\mathrm{b}} \mathrm{A}$ value of $b=5.0$ was assigned based on the general shape of the BDTL, though the parameter is not as critical to model convergence (subject to its constraints).

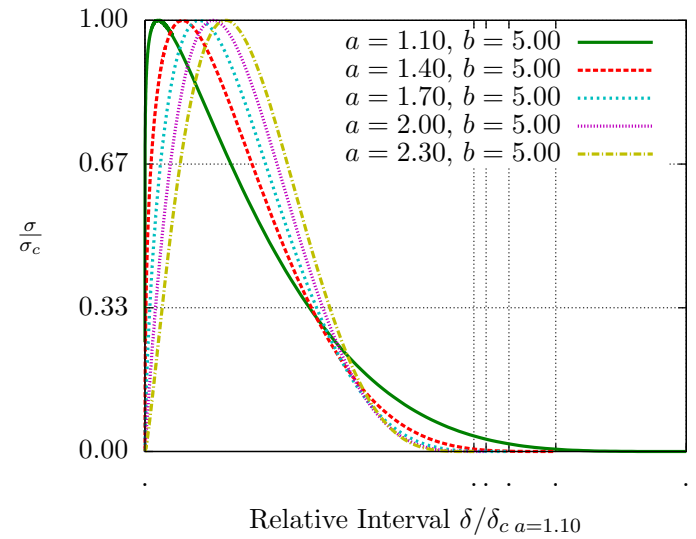

(a) The effect of a broad variation of the parameter $a$ on the normalized BDTL. Small values of $a$ skew the distribution to the left.

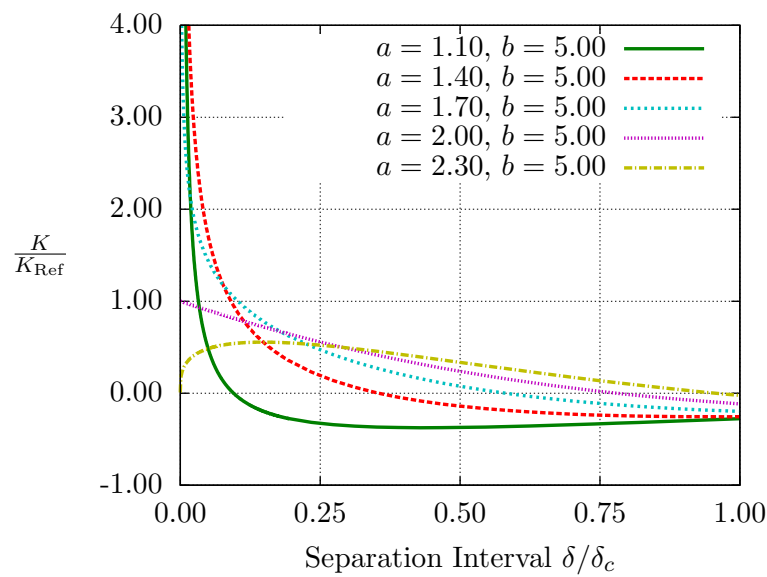

(c) The effect of a broad variation of the parameter $a$ on the (d) The effect of a narrow variation of the parameter $a \approx 2.0$ stiffness. The stiffness gradients are very large as parameter $a$ on the stiffness. Even a small departure from $a=2.0$ causes departs from 2.0.

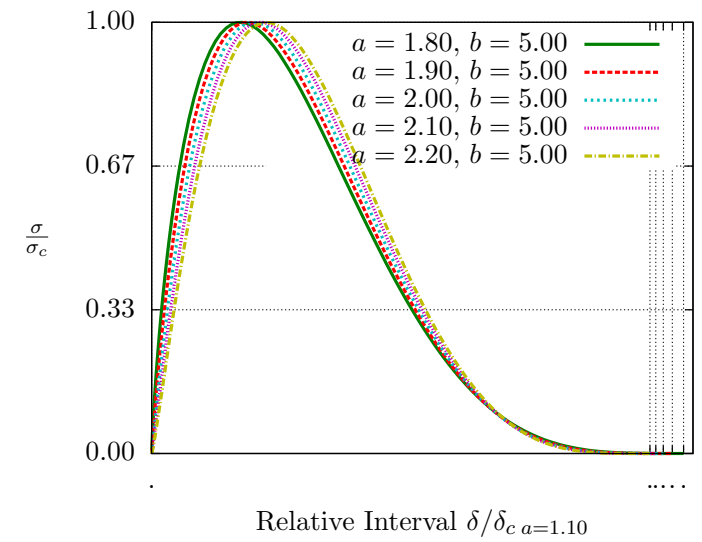

(b) The effect of a narrow variation of the parameter $a \approx$ 2.0 on the BDTL

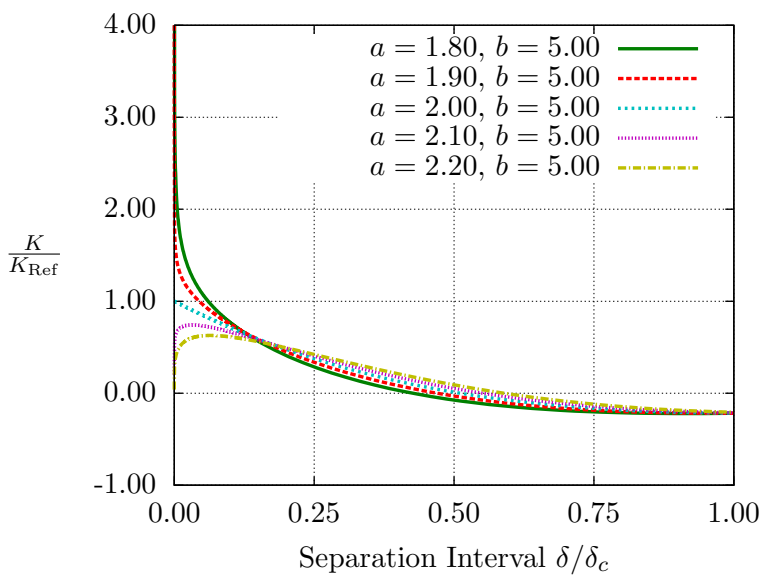

large stiffness gradients.

Figure 3: The effect of the parameter $a$ on the BDTL

\section{The sinusoidal traction law}

Although the BDTL is a much more smooth law than the TTL while resembling the shape of the TTL, it is certainly possible to conceive of a law that is more smooth. In the limit of obtaining a (non-zero) smooth stiffness derivative, a parabolic traction law could be assumed. Unfortunately, such an assumption would require that all cohesive integration points are simultaneously in zones where the stiffness is changing (since the resulting stiffness gradient would be a non-zero constant). Although the maximum stiffness

\footnotetext{
b This result could have be anticipated upon examining the form of the beta distribution equation, however the figures 3 (a)$3(d)$ provide an efficient means of interpretation.
} 
derivative would be minimized, the linearity assumptions underlying the Newton solver would be a poor global approximation of the response during every increment.

In a typical analysis, only a small portion of the cohesive integration points are in critical zones. The remainder of the cohesive elements are likely to be loaded with relatively low traction loads. Therefore, it is desirable for the assumed law to have regions of near linearity, particularly in the lower traction loads, such that only a relatively small number of critical integration points are undergoing a significant change in stiffness.

The BDTL provides this feature at some level, however the initial derivative of the stiffness (in the low traction region) is non-zero. A very simple function with a zero initial derivative and a low maximum derivative is the sine function. As a result, it may be a good function upon which to model a traction law.

As with all the traction laws, the integral of a sinusoidal stress-relative displacement curve must equal the critical energy release rate $\left(G_{\mathrm{c}}\right)$ and the maximum value must map to the critical stress $\left(\sigma_{\mathrm{c}}\right)$. The following traction law meets these requirements:

$$
\begin{aligned}
\sigma_{\beta}\left(\frac{\delta}{\delta_{\mathrm{c}}}\right) & =\sigma_{\mathrm{c}} \sin \left(\pi \frac{\delta}{\delta_{\mathrm{c}}}\right), \\
\delta_{\mathrm{c}} & =\frac{\pi G_{\mathrm{c}}}{2 \sigma_{\mathrm{c}}} .
\end{aligned}
$$

The stiffness at a given point takes the form of a constant times the cosine function and the second derivative remains relatively small as a constant times the sine function. The traction law in Eq. (18) is shown in figure 4.

The sinusoidal traction law (STL) provides a mathematically convenient formulation for a traction law. It does not resemble the commonly used laws, as it is symmetric about its midpoint and its initial stiffness is relatively low. However, the STL offers a clear advantage over the traditional laws. The initial stiffness derivative of the STL is zero while the maximum stiffness derivative is relatively small. Therefore, it is worth evaluating the efficiency of the STL.

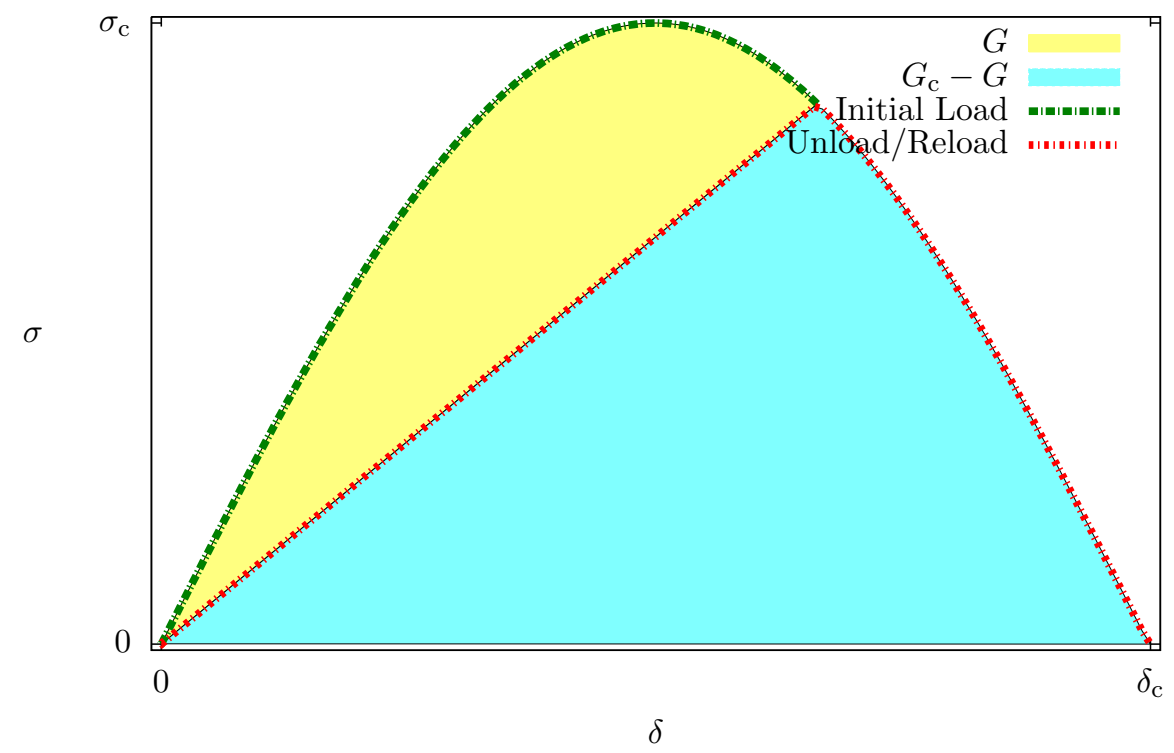

Figure 4: The sinusoidal traction law

\section{The DCZM FE models}

The core of the FE models in this work is the DCZM element of Gustafson and Waas. ${ }^{15}$ That element has been extended to include the traction laws described in section III. The DCZM element is illustrated (in 2D form) in figure 5 and conforms to the layout for a 2D four-node element in Abaqus®. A similar 3D version of 
element has also been developed. A typical peeling process zone associated with crack propagation is shown in figure 6 .

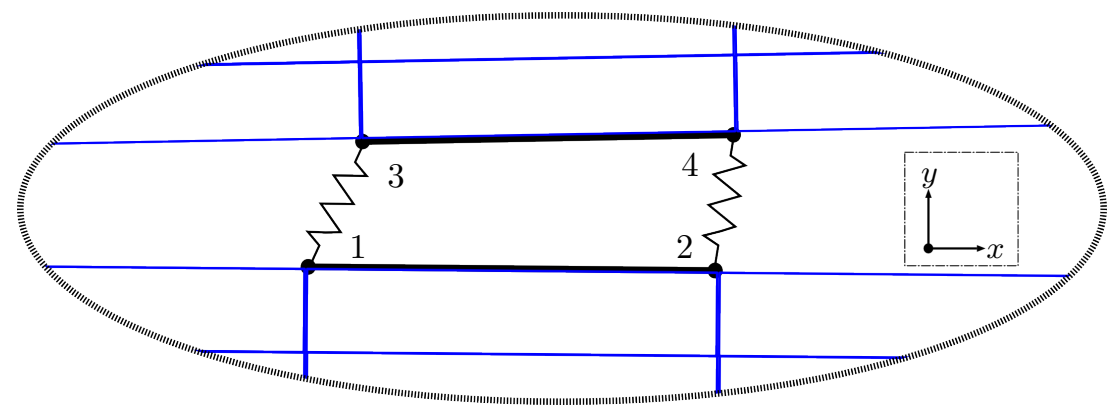

Figure 5: Four-node 2D DCZM element with surrounding elements. Adhesion is enforced with non-linear springs between node pairs.

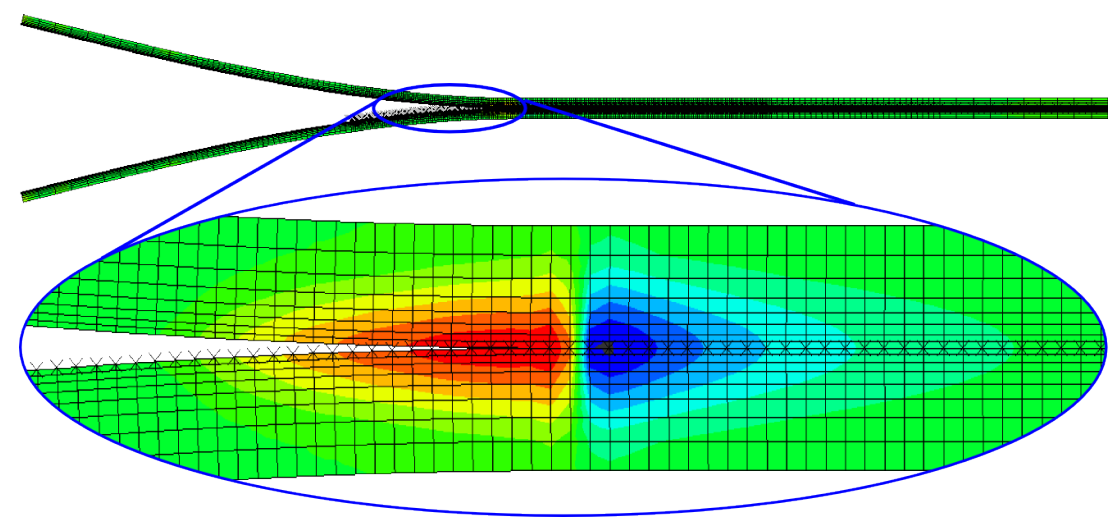

Figure 6: Typical process zone using the DCZM element

Two FE models were created for the efficiency and robustness comparison. The models are shown in figures 7 and 8. Each consists of linear plain strain elements (CPE4I) and the solver is Abaqus@ Standard. DCZM elements were used to model the adhesive layer. Abaqus ${ }^{\circledR}$ contact algorithms were used to manage post-failure contact and sliding in the end notch flexure (ENF) model. For each model, a minimum increment fraction of $10^{-15}$ was set and time incrementation controls were used. ${ }^{c}$ The purpose of the incrementation controls was to allow the solver to search beyond default levels for a deeper reliability assessment. Model size is reported in tables 1 and 2 .

Table 1: Approximate quantities in the SLJ FE model

\begin{tabular}{|l|r|}
\hline Number of elements & 7400 \\
Number of user nodes & 7900 \\
Number of variables & 51000 \\
\hline
\end{tabular}

\section{Comparison of traction law solution efficiency and robustness}

In brief summary of the section III, the form of the traction law affects the solution efficiency through the stiffness gradient as well as through its effect on $\delta_{\mathrm{c}}$. The critical stress is also a factor in solution efficiency through its effect on the value of $\delta_{\mathrm{c}}$.

\footnotetext{
${ }^{\mathrm{c}} *$ CONTROLS, PARAMETERS $=$ TIME INCREMENTATION

$4,8,18,32,10,10,24,10,12,10$
} 


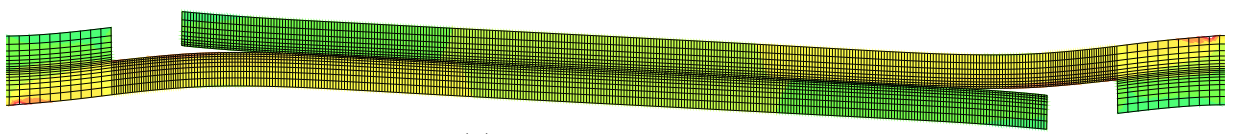

(a) A typical SLJ model

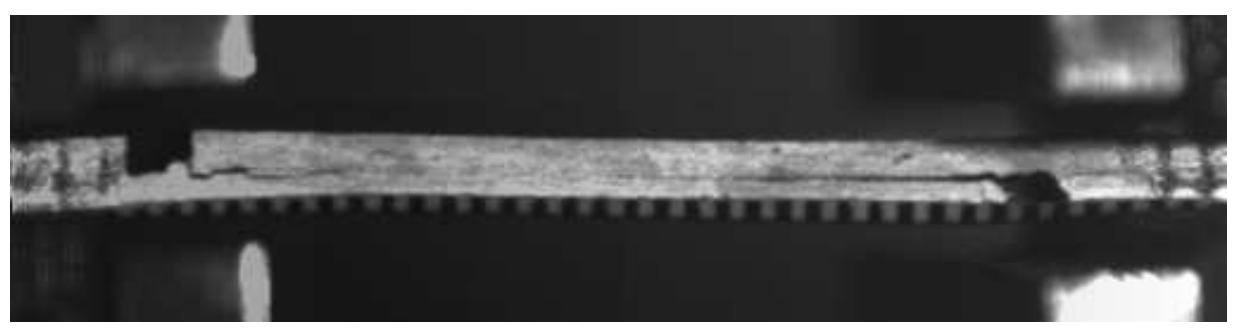

(b) A typical SLJ experiment

Figure 7: Typical SLJ model and experiment. These images were taken just prior to failure.

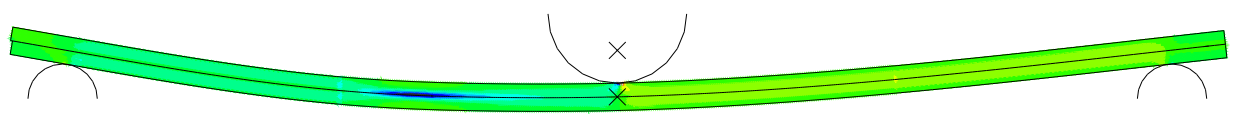

(a) Global view of the ENF model

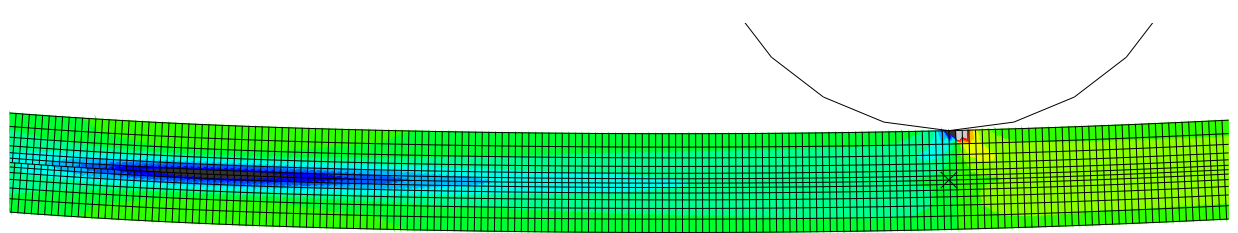

(b) Local view of the ENF model

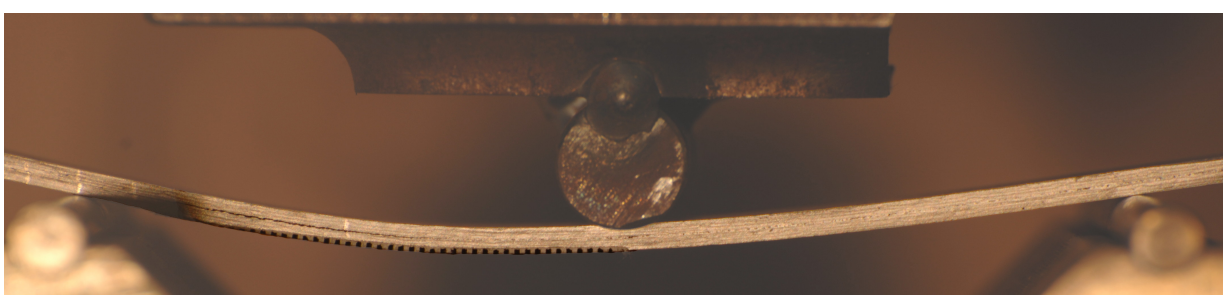

(c) A typical ENF experiment

Figure 8: Typical ENF model and experiment

Table 2: Approximate quantities in the ENF FE model

\begin{tabular}{|l|l|}
\hline Number of elements & 16000 \\
Number of user nodes & 14000 \\
Number of variables & 97000 \\
\hline
\end{tabular}


The purpose of the smooth laws is to improve the overall solution efficiency and robustness by minimizing the stiffness discontinuities and gradients. Whereas solver convergence and stability are well defined, solution efficiency is not well defined at the level of the constitutive law. Therefore, to evaluate the solution efficiency of the traction laws, it is useful to compare available metrics.

Two metrics for solution efficiency will be used to compare the traction laws. The first is the average size of a set of minimum size increments during a given solution. The stiffness gradient affects the likelihood of obtaining a converged solution and the minimum increment size is a simple metric which correlates to the ease of convergence. The second efficiency metric is the number of solver passes required to obtain a given solution. This is a reproducible metric of the CPU cost of a given solution. The number of solver passes will be identical across job repetition regardless of system resources, provided that the solver algorithm remains fixed. ${ }^{\mathrm{d}}$

Two efficiency metrics will be used since neither metric (on its own) is an adequate representation of the solution efficiency. For example, it is possible in an unstable solution (like the single lap joint) for a solver to converge without finding the peak load point. ${ }^{59}$ This is particularly true if large increments are maintained. As a result, a cap on increment size is often required in order to ensure that the peak load is found in a given model. Efficiency conclusions would be misleading if the peak load is not captured, however the cap adds a significant number of solver passes that would not otherwise be required. This makes the number of solver passes an imperfect metric of efficiency. On the other hand, the number of solver passes (the direct CPU cost) is not the same for each increment. A given increment could converge in one pass or tens of passes, making the increment size an imperfect metric of efficiency. Since neither metric is perfect, both will be used in the comparison.

The incrementally based solution efficiency is defined as the ratio of the mean increment size for the smallest ten increments $\left(\Delta t_{10}\right)$ to the maximum allowed increment size $\left(\Delta t_{\max }\right)$.

$$
\eta_{\text {Inc }}=\text { Mean } \frac{\Delta t_{10}}{\Delta t_{\max }}
$$

The smallest ten increments in a given analysis are exclusive of any step completion increments. $\Delta t_{\max }$ is specified to ensure that the peak load is captured. Ten increments are averaged in order to remove any isolated effects and to allow for some indication of increment size recovery.

The solver pass based solution efficiency is defined as:

$$
\eta_{\text {Pass }}=\frac{\Gamma_{\text {Min }}}{\Gamma_{\text {Actual }}}
$$

$\Gamma_{\text {Min }}$ is the minimum number of passes which would be required to solve the system (based on the specified size limits) and $\Gamma_{\text {Actual }}$ is the actual number of passes that were required. In both Eq. (19) and Eq. (20), the solution efficiency is set to zero if the job does not converge.

A comparison of the effective solution efficiency for a large set of single lap joint (SLJ) and ENF analyses were run with three ${ }^{e}$ traction laws. The two model types exhibit different failure mechanisms and global stabilities. The SLJ analysis exhibits catastrophic failure, however there is very little surface interaction once the cohesive bond has failed. The ENF analysis maintains global stability, however the surfaces remain in contact and continue to interact after adhesive failure. The two model types are representative of many of the applications of cohesive elements.

\section{A. Efficiency comparison}

For the SLJ models, a histogram of the solution efficiency based on minimum increment size is shown in figure 9(a). Figure 9(b) provides a similar comparison of the solver pass efficiency. Each is based on 1024 SLJ model runs. In the figures, the differences between the jobs within a given traction law are the governing parameters of the law $\left(G_{\text {IIc }}, \sigma_{\text {IIc }}\right.$, etc) and the geometry. ${ }^{\text {f }}$ The TTL models each have unique values of plasticity fraction $\left(\alpha_{\mathrm{pl}}\right)$, whereas the triangular law models all have zero plasticity (by definition). In all SLJ

\footnotetext{
d Alfano and Crisfield ${ }^{48,50}$ established a precedent for using the average increment size and the total number of iterations as metrics for convergence efficiency.

e The three laws are the trapezoidal traction law, the beta distribution traction law, and the sinusoidal traction law. In the plots of efficiency, the trapezoidal traction law is subdivided into a triangular law and the general trapezoidal law.

$\mathrm{f}$ The kriging analysis sites from Gustafson and Waas ${ }^{60}$ are used to set an array of parameter values for the efficiency comparison.
} 
analyses, a viscous damping coefficient ${ }^{g}$ of $\mu=10^{4}$ was used to assist in convergence due to the catastrophic failure associated with the test.

From the SLJ pass efficiency results $\left(\eta_{\text {Pass }}\right)$, it is apparent that the smooth traction laws require fewer solver passes (on average) than does the TTL. However, the overall effect of the traction law on the minimum increment efficiency $\left(\eta_{\text {Inc }}\right)$ is inconclusive as there are no definitive trends present in the data. It is likely that the catastrophic failure mode of the SLJ test drives the minimum increment size downwards at the point of failure and that the success of viscous stabilization is quasi-random among the different models. Interestingly, the triangular and general forms of the trapezoidal traction law have nearly identical convergence characteristics for the SLJ test.

A more definitive result is found in the ENF model efficiency. Figures 9(c) and 9(d) report minimum increment and solver pass efficiencies for $1024 \mathrm{ENF}$ model runs. As with the SLJ models, the difference between the models within a law are the values of the adhesive parameters $\left(G_{\text {IIc }}, \sigma_{\text {IIc }}\right.$, etc $)$ and the geometry. In this set of figures, it is clear that the smooth traction laws outperform the laws based on the TTL. The minimum increment size remains larger and the number of solver passes is smaller for the smooth laws. Among the triangular and trapezoidal forms of the TTL, there is a negligible difference in performance. In comparing the smooth laws, the STL clearly outperforms the BDTL in both metrics of efficiency.

\section{B. Robustness comparison}

A final metric of traction law performance is the overall ability of the solution to converge for an analysis type. Key to this metric is the reliability of convergence. If a traction law is very fast for some analyses but fails to converge for other analyses, then the law is non-optimal. An analyst is likely to choose a more reliable law with a cost that is somewhat higher than a cheap law that is suspect with respect to convergence reliability.

Table 3 reports the percentage of jobs that were successfully completed for the two model types. Using the TTL as the baseline, the BDTL was slightly less reliable and the STL was significantly more reliable for the SLJ model type. The ENF model type was more definitive as the BDTL and STL were both very robust in comparison to the TTL. More than twice as many jobs were successfully completed when the smooth laws were used than when the TTL law was used. Of all the traction laws, the STL was the most reliable in both model types by a relatively large margin.

Table 3: Percentage of jobs that converged to completion

\begin{tabular}{|l|c|c|c|c|}
\hline & Triangle & TTL & BDTL & STL \\
\hline SLJ & $65.9 \%$ & $65.9 \%$ & $61.4 \%$ & $80.7 \%$ \\
ENF & $29.8 \%$ & $25.0 \%$ & $61.7 \%$ & $76.7 \%$ \\
\hline
\end{tabular}

\section{Conclusion}

A DCZM finite element and its associated traction laws have been evaluated from the perspective of efficiency and robustness for use in modeling decohesion in structural applications. Three traction laws are presented, one which is commonly used and the others which are developed with computational efficiency and robustness in mind.

A comparison of the efficiency of the three laws has been shown in the context of their application to two coupon-level experiments. In both model types, the smooth laws (the beta distribution traction law and the sinusoidal traction law) have been shown to reduce the number of solver passes required to converge to a completed solution. The effect of traction law on the minimum increment size is mixed. The smooth laws have a clear net positive effect on the minimum increment size in the ENF models, whereas in the SLJ model they do not have a clear positive or negative effect. The global instability of the SLJ structure likely dominates this result, driving the minimum increment size down in both model types. Of the two efficiency metrics, the number of solver passes is the most direct metric of computation cost. The efficiency based on

${ }^{\mathrm{g}}$ Damping is managed internally by the DCZM element ${ }^{15}$ and is enforced only on the relative displacements of the cohesive element nodes. 


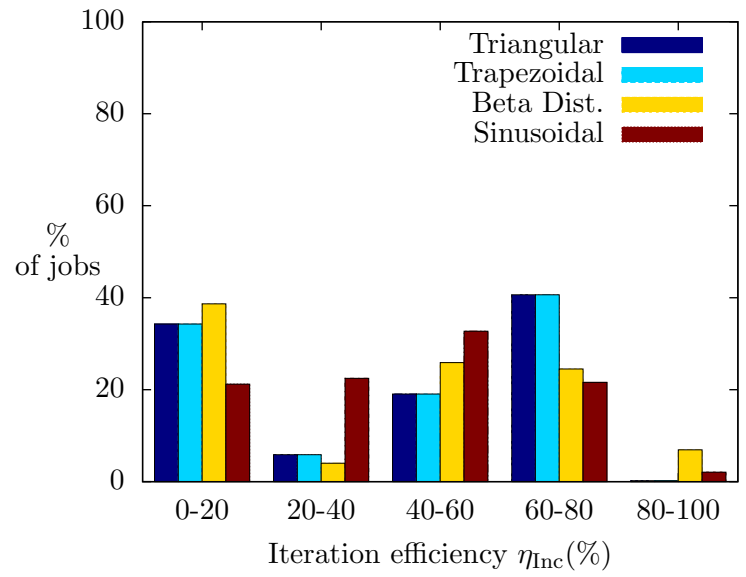

(a) A measure of efficiency for SLJ models based on increment size. The $\eta_{\text {Inc }}$ metric is inconclusive for this model type.

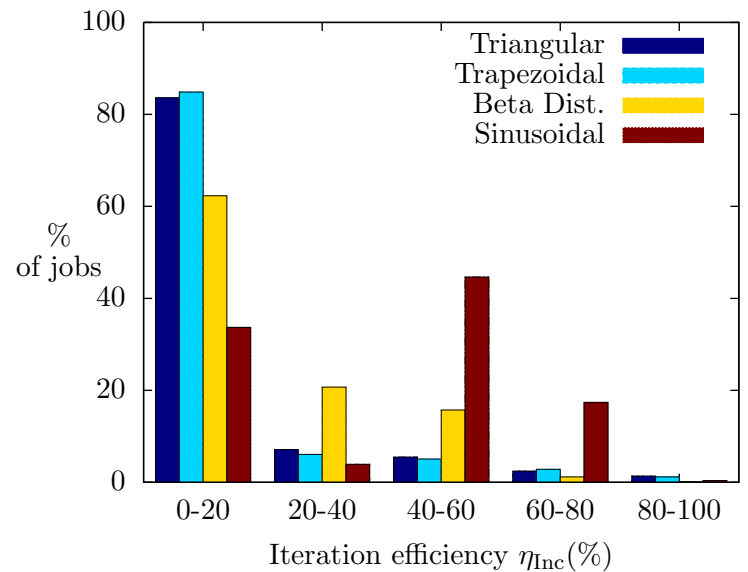

(c) A measure of efficiency for ENF models based on increment size. The smooth laws are significantly more efficient based on the $\eta_{\text {Inc }}$ metric for this model type.

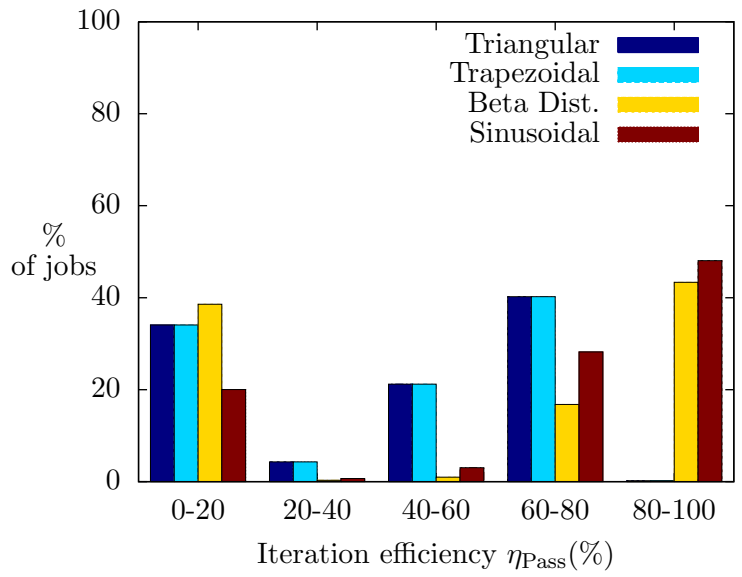

(b) A measure of efficiency for SLJ models based on the number of solver passes. The smooth laws are significantly more efficient based on the $\eta_{\text {Pass }}$ metric for this model type.

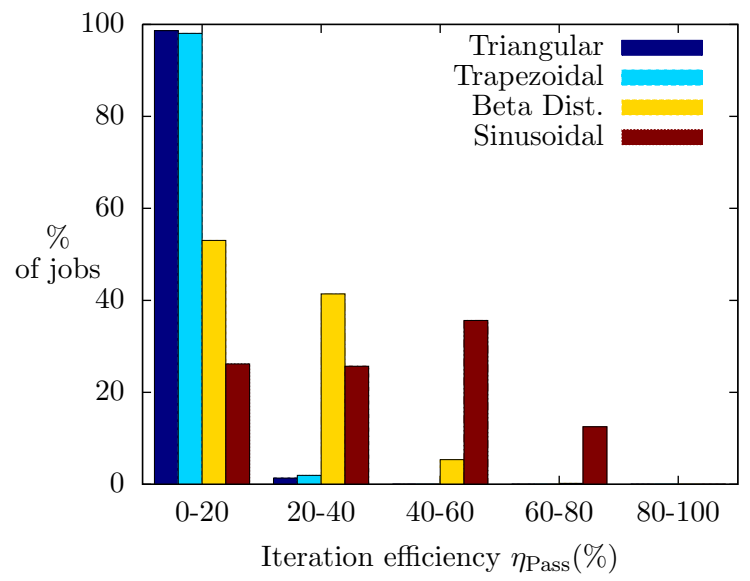

(d) A measure of efficiency for ENF models based on the number of solver passes. The smooth laws are significantly more efficient based on the $\eta_{\text {Pass }}$ metric for this model type.

Figure 9: Model efficiency 
minimum increment size is a useful metric primarily when the number of solver passes is influenced by other modeling requirements.

The choice of traction law was found to have a significant effect on the overall solution robustness. SLJ models based on the BDTL were found to be slightly less likely to complete than the TTL whereas SLJ models based on the STL were found to be more likely to complete. For ENF, models with either of the smooth traction laws were significantly more likely to obtain a converged solution than models with the TTL. Of the three laws, the STL was found to be the most efficient and the most robust. The BDTL is more efficient than the TTL, but robustness depends on the problem being solved.

With a general trend towards improved convergence and robustness resulting from use of the smooth laws, it is shown that the form of the traction law should be considered when modeling adhesively bonded structures. The use of a smooth law is likely to reduce the overall cost of computation without effecting the global response or accuracy of the solution.

\section{A. List of acronyms}

BDTL beta distribution traction law

CCZM continuous cohesive zone method

CPU central processing unit

DCZM discrete cohesive zone method

ENF end notch flexure

FE finite element

PDF probability density function

SLJ single lap joint

STL sinusoidal traction law

TTL trapezoidal traction law

VCCT virtual crack closure technique

CPE4I linear plain strain elements

\section{Acknowledgments}

This work was supported by the Space Vehicle Technology Institute under grant NCC3-989 jointly funded by NASA and the Department of Defense. It is managed within the NASA Constellation University Institutes Project, with Claudia Meyer as the project manager and H. Kevin Rivers and Stanley Smeltzer as the project monitors.

\section{References}

${ }^{1}$ Wooley, G. R. and Carver, D. R., "Stress Concentration Factors for Bonded Lap Joints," J. Aircraft, Vol. 8, 1971, pp. $817-820$.

${ }^{2}$ Adams, R. D. and Peppiatt, N. A., "Stress Analysis of Adhesive-Bonded Lap Joints," J. Strain Analysis, Vol. 9, No. 3, 1974, pp. 185-196.

${ }^{3}$ Kafkalidis, M. S. and Thouless, M. D., "The effects of geometry and material properties on the fracture of single lap-shear joints," Int J Solids Struct, Vol. 39, No. 17, Aug. 2002, pp. 4367-4383.

${ }^{4}$ Xie, D., Waas, A. M., Shahwan, K. W., Schroeder, J. A., and Boeman, R. G., "Fracture criterion for kinking cracks in a tri-material adhesively bonded joint under mixed mode loading," Eng Fract Mech, Vol. 72, No. 16, Nov. 2005, pp. $2487-2504$.

${ }^{5} \mathrm{Li}, \mathrm{S}$. , Thouless, M., Waas, A., Schroeder, J., and Zavattieri, P., "Use of a cohesive-zone model to analyze the fracture of a fiber-reinforced polymer-matrix composite," Compos Sci Technol, Vol. 65, No. 3-4, March 2005, pp. 537-549.

${ }^{6} \mathrm{Li}$, S., Thouless, M., Waas, A., Schroeder, J., and Zavattieri, P., "Competing failure mechanisms in mixed-mode fracture of an adhesively bonded polymer-matrix composite," Int. J. Adhes. Adhes., Vol. 26, No. 8, Dec. 2006, pp. 609-616. 
${ }^{7}$ Valoroso, N. and Champaney, L., "A damage-mechanics-based approach for modelling decohesion in adhesively bonded assemblies," Eng Fract Mech, Vol. 73, No. 18, Dec. 2006, pp. 2774-2801.

${ }^{8}$ Hillerborg, A., Modeer, M., and Petersson, P., "Analysis of crack formation and crack growth in concrete by means of fracture mechanics and finite elements," Cem. Concr. Res., Vol. 6, 1976, pp. 773-782.

${ }^{9}$ Song, S. J. and Waas, A. M., "Mode I failure of laminated polymeric composites," Eng Fract Mech, Vol. 49, No. 1, 1994, pp. $17-27$.

${ }^{10}$ Song, S. J. and Waas, A. M., "Energy-based mechanical model for mixed mode failure of laminated composites," $A I A A$ $J$, Vol. 33, No. 4, 1995, pp. 739-745.

${ }^{11}$ Borg, R., Nilsson, L., and Simonsson, K., "Simulation of delamination in fiber composites with a discrete cohesive failure model," Composites Science and Technology, Vol. 61, No. 5, April 2001, pp. 667-677.

${ }^{12}$ Borg, R., Nilsson, L., and Simonsson, K., "Modeling of delamination using a discretized cohesive zone and damage formulation," Composites Science and Technology, Vol. 62, No. 10-11, Aug. 2002, pp. 1299-1314.

${ }^{13}$ Xie, D. and Waas, A. M., "Discrete cohesive zone model for mixed-mode fracture using finite element analysis," Eng Fract Mech, Vol. 73, No. 13, Sept. 2006, pp. 1783-1796.

${ }^{14}$ Xie, D., Salvi, A. G., Sun, C., Waas, A. M., and Caliskan, A., "Discrete Cohesive Zone Model to Simulate Static Fracture in 2D Triaxially Braided Carbon Fiber Composites," Journal of Composite Materials, 2006.

${ }^{15}$ Gustafson, P. A. and Waas, A. M., "T650/AFR-PE-4/FM680-1 Mode I Critical Energy Release Rate at High Temperatures: Experiments and Numerical Models," Proceedings of the AIAA/ASME/ASCE/AHS/ASC 48th Structures, Structural Dynamics, and Materials Conference, Apr 23-26 2007, Honolulu HI, No. 2007-2305, American Institute of Aeronautics and Astronautics, 2007.

${ }^{16}$ Gillespie, Jr, J. W., Carlsson, L. A., and Pipes, R. B., "Finite element analysis of the end notched flexure specimen for measuring mode II fracture toughness," Compos Sci Technol, Vol. 27, No. 3, 1986, pp. 177-197.

${ }^{17}$ Wang, J. T., Raju, I. S., and Sleight, D. W., "Fracture mechanics analyses of composite skin-stiffener debond configurations with shell elements," No. 94-1389-CP, 1994.

${ }^{18}$ Glaessgen, E., Raju, I., and C.C. Poe, J., "Fracture mechanics analysis of stitched stiffener-skin debonding," 39th AIAA/ASME/ASCE/AHS/ASC Structures, Structural Dynamics, and Materials Conference, No. 98-2022, 1998.

${ }^{19}$ Krueger, R., "Virtual crack closure technique: History, approach, and applications," Applied Mechanics Reviews, Vol. 57, No. 2, 2004, pp. 109-143.

${ }^{20}$ Xie, D., Waas, A. M., Shahwan, K. W., Schroeder, J. A., and Boeman, R. G., "Computation of Energy Release Rates for Kinking Cracks based on Virtual Crack Closure Technique," Computer Modeling in Engineering E5 Sciences, Vol. 6, 2004, pp. $515-524$.

${ }^{21}$ Xie, D., Salvi, A. G., Waas, A. M., and Caliskan, A., "Discrete cohesive zone model to simulate static fracture in carbon fiber textile composites," 46th AIAA/ASME/ASCE/AHS/ASC Structures, Structrual Dynamics and Materials Conference, 2005.

${ }^{22}$ Xie, D. and Biggers, Jr, S. B., "Progressive crack growth analysis using interface element based on the virtual crack closure technique," Finite Elem. Anal. Des., Vol. 42, No. 11, 2006, pp. 977-984.

${ }^{23}$ de Borst, R., "Numerical aspects of cohesive-zone models," Eng Fract Mech, Vol. 70, No. 14, Sept. 2003, pp. $1743-1757$.

${ }^{24}$ Munoz, J., Galvanetto, U., and Robinson, P., "On the numerical simulation of fatigue driven delamination with interface elements," Int J Fatigue, Vol. 28, No. 10, Oct. 2006, pp. 1136-1146.

${ }^{25}$ Goncalves, J. P. M., de Moura, M. F. S. F., and de Castro, P. M. S. T., "A three-dimensional finite element model for stress analysis of adhesive joints," Int. J. Adhes. Adhes., Vol. 22, No. 5, 2002, pp. 357-365.

${ }^{26}$ Goyal, V. K., Johnson, E. R., and Cassino, C., "Computational model for progressive failure of adhesivley bonded joints," 44 th AIAA/ASME/ASCE/AHS/ASC Structures, Structrual Dynamics and Materials Conference, 2003.

${ }^{27}$ Davies, G., Hitchings, D., and Ankersen, J., "Predicting delamination and debonding in modern aerospace composite structures," Compos Sci Technol, Vol. 66, No. 6, May 2006, pp. 846-854.

${ }^{28}$ Davidson, B. D., Gharibian, S. J., and Yu, L., "Evaluation of energy release rate-based approaches for predicting delamination growth in laminated composites," Int J Fracture, Vol. V105, No. 4, Oct. 2000, pp. 343-365.

${ }^{29}$ Pietruszczak, S. and Mroz, Z., "Finite element analysis of deformation of strain-softening materials," Int J Numer Meth Eng, Vol. 17, No. 3, 1981, pp. 327-334.

${ }^{30}$ Ungsuwarungsri, T. and Knauss, W., "The role of damage-softened material behavior in the fracture of composites and adhesives," Int J Fracture, Vol. 35, No. 3, 1987, pp. 221-241.

${ }^{31}$ Tvergaard, V. and Hutchinson, J., "The Relation Between Crack Growth Resistance and Fracture Process Parameters in Elastic-Plastic Solids," Journal of the Mechanics and Physics of Solids(UK), Vol. 40, No. 6, 1992, pp. 1377-1397.

${ }^{32}$ Schellekens, J. and de Borst, R., "On the numerical integration of interface elements," Int J Numer Meth Eng, Vol. 36, No. 43-66, 1993, pp. 30-31.

${ }^{33} \mathrm{Xu}, \mathrm{X}$. and Needleman, A., "Numerical simulations of fast crack growth in brittle solids," J. Mech. Phys. Solids, Vol. 42, No. 9, 1994, pp. 1397-1407.

${ }^{34}$ Camacho, G. and Ortiz, M., "Computational modelling of impact damage in brittle materials," Int J Solids Struct, Vol. 33, No. 20, 1996, pp. 2899-2938.

${ }^{35}$ Simulia, I., Abaqus User Manual v6.7, Electronic Version, 2007.

${ }^{36}$ Camanho, P. P. and Davila, C. G., "Mixed-Mode Decohesion Finite Elements for the Simulation of Delamination in Composite Materials," Tech. Rep. TM-2002-211737, NASA, 2002.

${ }^{37}$ Alpha STAR Corp., GENOA, 5199 East Pacific Coast Highway, Suite 410 Long Beach, California 90804 USA, 2008.

${ }^{38}$ Sandia National Laboratory, Tahoe User Guide, 3rd ed., May 2003.

${ }^{39}$ Albouyso, V., Allix, O., Ladeveze, P., and Leveque, D., "Interfacial approach of delamination: possibilities and difficulties," Proceedings of the 12th International Conference on Composite Materials, Paris, France., 1999. 
${ }^{40}$ de Borst, R., "Some recent issues in computational failure mechanics," Int. J. Numer. Meth. Engng, Vol. 52, No. 5, 2001, pp. 63-96.

${ }^{41}$ Zhou, F. and Molinari, J., "Dynamic crack propagation with cohesive elements: a methodology to address mesh dependency," Int. J. Numer. Meth. Engng, Vol. 59, 2004, pp. 1-24.

${ }^{42}$ Oterkus, E., Barut, A., Madenci, E., Smeltzer, S., and Ambur, D., "Nonlinear analysis of bonded composite single-lap joints," Proceedings of the AIAA/ASME/ASCE/AHS/ASC 45th Structures, Structural Dynamics, and Materials Conference, April 19-22, Palm Springs, CA, No. 2004-1560, American Institute of Aeronautics and Astronautics, 2004.

${ }^{43}$ Oterkus, E., Madenci, E., Smeltzer, S., and Ambur, D., "Nonlinear analysis of bonded composite tubular lap joints," Proceedings of the AIAA/ASME/ASCE/AHS/ASC 46th Structures, Structural Dynamics, and Materials Conference, April 18-21, Austin, TX, No. 2005-2380, American Institute of Aeronautics and Astronautics, 2005.

${ }^{44}$ Smeltzer, S. S. and Lundgren, E., "Analytical and Numerical Results for an Adhesively Bonded Joint Subjected to Pure Bending," Proceedings of the AIAA/ASME/ASCE/AHS/ASC 47th Structures, Structural Dynamics, and Materials Conference, May 1-4 2006, Newport RI, No. 2006-1960, American Institute of Aeronautics and Astronautics, 2006.

${ }^{45} \mathrm{Cui}, \mathrm{W}$. and Wisnom, M., "A combined stress-based and fracture-mechanics-based model for predicting delamination in composites," Composites, Vol. 24, No. 6, 1993, pp. 467-474.

${ }^{46}$ Shahwan, K. and Waas, A., "Non-self-similar decohesion along a finite interface of unilaterally constrained delaminations," Proceedings: Mathematical, Physical and Engineering Sciences, Vol. 453, No. 1958, 1997, pp. 515-550.

${ }^{47}$ Davies, G. A. O., Robinson, P., Robson, J., and Eady, D., "Shear driven delamination propagation in two dimensions," Composites Part A: Applied Science and Manufacturing, Vol. 28, No. 8, June 1997, pp. 757-765.

${ }^{48}$ Alfano, G. and Crisfield, M. A., "Finite element interface models for the delamination analysis of laminated composites: mechanical and computational issues," Int J Numer Meth Eng, Vol. 50, No. 7, 2001, pp. 1701-1736.

${ }^{49} \mathrm{Mi}$, Y., Crisfield, M. A., Davies, G. A. O., and Hellweg, H. B., "Progressive Delamination Using Interface Elements," Journal of Composite Materials, Vol. 32, No. 14, July 1998, pp. 1246-1272.

${ }^{50}$ Alfano, G. and Crisfield, M., "Solution strategies for the delamination analysis based on a combination of local-control arc-length and line searches," Internat. J. Numer. Methods Eng, Vol. 58, 2003, pp. 999-1048.

${ }^{51}$ Corigliano, A., Mariani, S., and Pandolfi, A., "Numerical modeling of rate-dependent debonding processes in composites," Compos Struct, Vol. 61, No. 1-2, July 2003, pp. 39-50.

${ }^{52}$ Reedy Jr, E., Mello, F., and Guess, T., "Modeling the Initiation and Growth of Delaminations in Composite Structures," Journal of Composite Materials, Vol. 31, No. 8, 1997, pp. 812.

${ }^{53}$ Pandolfi, A., Krysl, P., and Ortiz, M., "Finite element simulation of ring expansion and fragmentation: The capturing of length and time scales through cohesive models of fracture," Int J Fracture, Vol. 95, No. 1, 1999, pp. 279-297.

${ }^{54}$ Espinosa, H. D., Zavattieri, P. D., and Dwivedi, S. K., "A finite deformation continuum discrete model for the description of fragmentation and damage in brittle materials," J Mech Phys Solids, Vol. 46, No. 10, Oct. 1998, pp. 1909-1942.

${ }^{55}$ Zavattieri, P. D., Raghuram, P. V., and Espinosa, H. D., "A computational model of ceramic microstructures subjected to multi-axial dynamic loading," J Mech Phys Solids, Vol. 49, No. 1, Jan. 2001, pp. 27-68.

${ }^{56} \mathrm{Li}$, S., Thouless, M., Waas, A., Schroeder, J., and Zavattieri, P., "Mixed-mode cohesive-zone models for fracture of an adhesively bonded polymer-matrix composite," Eng Fract Mech, Vol. 73, No. 1, Jan. 2006, pp. 64-78.

${ }^{57}$ Gustafson, P. A., Analytical and Experimental Methods for Adhesively Bonded Joints Subjected to High Temperatures, Ph.D. thesis, University of Michigan, 2008.

${ }^{58}$ Roudolff, F. and Ousset, Y., "Comparison between two approaches for the simulation of delamination growth in a D.C.B. specimen," Aerospace Science and Technology, Vol. 6, No. 2, Feb. 2002, pp. 123-130.

${ }^{59}$ Chaboche, J. L., Feyel, F., and Monerie, Y., "Interface debonding models: a viscous regularization with a limited rate dependency," Int J Solids Struct, Vol. 38, No. 18, May 2001, pp. 3127-3160.

${ }^{60}$ Gustafson, P. A. and Waas, A. M., "The interactions of adhesive constitutive parameters and their effects on common adhesive experiments," Internation Journal of Solids and Structures, 2008, Submitted. 